\title{
Modelling the Electromagnetic Separation of Non-metallic Particles from Liquid Metal Flowing through a Two-stage Multichannel
}

\author{
Da SHU, ${ }^{1)}$ J. MI, ${ }^{2)}$ Jun WANG ${ }^{1)}$ and Baode SUN"1 \\ 1) State Key Laboratory of Metal Matrix Composites, Shanghai Jiao Tong University, No. 800 Dongchuan Road, Shanghai \\ 200240 China. $\quad$ 2) Department of Materials, University of Oxford, Parks Road, Oxford OX1 3PH, UK.
}

(Received on July 14, 2010; accepted on September 24, 2010)

\begin{abstract}
A two-stage multichannel was designed to increase the efficiency of separating non-metallic particles from liquid metal flowing through an alternating magnetic field. Numerical method was developed to calculate the particle concentration and separation efficiency of a zinc melt containing dross particles and verified by the experimental results. The distribution of particle concentration and axial fluid velocity changed significantly due to the added walls in the sub-channel, resulting in an abrupt increase in the residence time of the inner bulk melt with high particle concentrations and a remarkable increase in particle separation efficiency when flowing through the single-channel to sub-channels. A multistage and multichannel arrangement is hence recommended for further increase in the separation efficiency of an electromagnetic separator.
\end{abstract}

KEY WORDS: modelling; electromagnetic separation; non-metallic particles; zinc dross.

\section{Introduction}

For high quality premium metallic alloys in critical applications e.g. aerospace structural components and electronic devices, non-metallic particles (inclusions) present in the materials often cause serious problems during services, such as stress concentration, impairment in strength and ductility, ${ }^{1)}$ toughness degradation, ${ }^{2)}$ and poor surface finish. Therefore, it is always important to separate and remove such non-metallic particles from the matrix materials especially during the liquid state prior to cast into a final product.

Electromagnetic separation-a method of separating non-metallic inclusions from liquid metal by imposing an electromagnetic field to the channel or pipe (termed separator hereafter) through which the liquid metal flows-has been identified as a generic technology for the production of ultra-clean metals ${ }^{3}$ ) because this process can be easily implemented by controlling the magnitude and direction of an imposed electromagnetic force. Laboratory-scale experiments have demonstrated successfully the separation of (i) non-metallic inclusions $\left(\mathrm{Al}_{2} \mathrm{O}_{3}{ }^{4}\right)$ or $\mathrm{SiC}^{5)}$ ) from $\mathrm{Al}$ melt, (ii) Fe-rich phase from hypoeutectic Al-Si alloys, ${ }^{3,6)}$ primary $\mathrm{Si}$ particles from hypereutectic Al-Si alloys, ${ }^{7)}$ and $\mathrm{Fe}-\mathrm{Al}-\mathrm{Zn}$ dross from galvanizing $\mathrm{Zn}$ melt, ${ }^{8,9)}$ where a single straightpass circular or square separator with a uniform cross-section are commonly used. For industrial applications, the feasibility of using a bundle of small channels for inclusion removal has also been investigated. ${ }^{10)}$

However, for an alternating magnetic field, weak force density in the central region of a bulk melt is unavoidable due to the inherent skin effect of high frequency magnetic field, ${ }^{11)}$ making it difficult for the particles initially inside the central region of a separator or channel to be moved towards the wall. Thus the efficiency of separating inclusions from liquid metal flowing through a single straight-pass separator is limited, especially in the case of high liquid metal flow rate. This paper presents a novel method to increase the efficiency of separating inclusions by using a multistage multichannel separator i.e. dividing a single channel to sub-channels in the flow direction. A numerical method has been developed to simulate the flow field in a single-square and a two-stage multi-square separator, and the particle concentration and separation efficiency of dross particles from zinc melt flowing through both separator arrangements. The main reason for improvement in separation efficiency by the multi-square separator is discussed and comparison with the corresponding experiments is also presented.

\section{Experimental}

Figure 1 shows schematically the experimental appara-

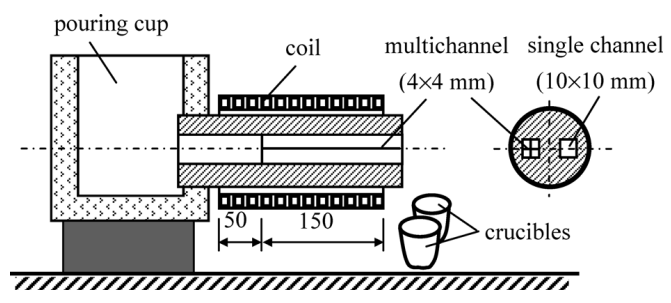

Fig. 1. A schematic view of the experimental apparatus. 
tus, consisting of a pouring cup, a copper coil and a cordierite $\left(2 \mathrm{MgO} \cdot \mathrm{Al}_{2} \mathrm{O}_{3} \cdot 5 \mathrm{SiO}_{2}\right)$ ceramic separator with two channels: a single-square channel of uniform cross-section of $10 \mathrm{~mm} \times 10 \mathrm{~mm}$, and a two-stage multichannel which has the same cross-section as the single-square channel at the front $(50 \mathrm{~mm})$ and four sub-channels of cross-section of $4 \mathrm{~mm} \times 4 \mathrm{~mm}$ each at the rear three-quarter $(150 \mathrm{~mm})$ of the flow pass inside the coil. The total length of the separator is $280 \mathrm{~mm}$ and the wall thickness between sub-channels is $1 \mathrm{~mm}$. The copper coil has a dimension of $75 \mathrm{~mm}$ (inner diameter) $\times 200 \mathrm{~mm}$ (length) and was powered by an induction power supply with a capability of producing alternating currents at a frequency of $17.5 \mathrm{kHz}$ and a maximum effective magnetic flux density of $0.05 \mathrm{~T}$ in the coil.

Pure zinc (99.995 wt\%) ingots were melted in an electrical resistance furnace to $700^{\circ} \mathrm{C}$ and added with trace $(\sim 0.1 \mathrm{wt} \%)$ low alloy high strength cold rolling steel and excessive $(1.0 \mathrm{wt} \%)$ high purity aluminum $(99.999 \mathrm{wt} \%)$ to generate $\mathrm{Fe}_{2} \mathrm{Al}_{5}$ intermetallic compounds - known as float dross $^{12)}$ that impairs the surface quality of the steel strip in a commercial continuous galvanizing line due to excessive dissolution of $\mathrm{Fe}$ above its solubility. The melt was cooled to $480^{\circ} \mathrm{C}$ and held for $2 \mathrm{~h}$ before poured into the pouring cup, and flowed through the single channel and the twostage multichannel simultaneously, then collected by two crucibles at the other end, respectively. The metal flow rate was controlled manually and estimated to be about $1.3 \mathrm{~kg} / \mathrm{min}$ or $33 \mathrm{~mm} / \mathrm{s}$. Metallographic samples were taken from the furnace before pouring, the crucibles and the residues remained in the multichannel after processing respectively. The specimens were sectioned, mounted and polished for observation using an optical microscope. The average area fraction and size distribution of particles over 40 fields of view for each specimen were measured using an image analysis system to evaluate the separation efficiency of dross particles:

$$
\eta_{1}=\left(1-\frac{s_{1}}{s_{0}}\right) \times 100 \%
$$

where $s_{0}$ and $s_{1}$ are the measured average area fraction of the particles in the specimen before and after processing respectively.

The weight percentage of $\mathrm{Fe}$ in the specimens, $C_{\mathrm{Fe}}$, was analysed using an Iris Advantage 1000 inductively Coupled Plasma-Atomic Emission Spectrometry method (ICPAES). Because almost all $\mathrm{Fe}$ in the alloy reacted with $\mathrm{Al}$ and formed $\mathrm{Fe}_{2} \mathrm{Al}_{5}$ dross due to excessive addition of $\mathrm{Al}$ according to $\mathrm{Zn}-\mathrm{Fe}-\mathrm{Al}$ ternary phase diagram, the weight percentage of the dross, $C_{\mathrm{D}}$, was calculated by:

$$
C_{\mathrm{D}}=\frac{2 \times 56+5 \times 27}{2 \times 56} \cdot C_{\mathrm{Fe}}=2.2 C_{\mathrm{Fe}}
$$

where 56 and 27 are the atomic weight of $\mathrm{Fe}$ and $\mathrm{Al}$ respectively.

The separation efficiency can also be expressed by the change of dross concentration:

$$
\eta_{2}=\left[1-\frac{\left(C_{\mathrm{D}}\right)_{1}}{\left(C_{\mathrm{D}}\right)_{0}}\right] \times 100 \%
$$

where $\left(C_{\mathrm{D}}\right)_{0}$ and $\left(C_{\mathrm{D}}\right)_{1}$ are the weight percentage of the dross in the specimen before and after processing respectively.

\section{Modelling}

Figure 2 shows the computational domain for the twostage multichannel in an infinitely long solenoid coil, where liquid metal flowed through the separator with an axial velocity, $w$. By taking the advantage of axis symmetry, a quarter cross-section of the large single-square channel and one full section of the sub-channels were chosen as the computational domain and discretised by $30 \times 30$ equal-size square meshes. A pseudo 3-D model ${ }^{11)}$ based on Taniguchi's model ${ }^{13)}$ was used to calculate the flow field in the large single-channel and sub-channel and the governing equations are:

$$
\begin{aligned}
& \frac{\partial u}{\partial x}+\frac{\partial v}{\partial y}=0 \\
& \rho_{f} u \frac{\partial u}{\partial x}+\rho_{f} v \frac{\partial u}{\partial y}=-\frac{\partial u}{\partial x}+f_{x}+\mu_{f}\left(\frac{\partial^{2} u}{\partial x^{2}}+\frac{\partial^{2} u}{\partial y^{2}}\right) \\
& \rho_{f} u \frac{\partial v}{\partial x}+\rho_{f} v \frac{\partial v}{\partial y}=-\frac{\partial p}{\partial y}+f_{y}+\mu_{f}\left(\frac{\partial^{2} v}{\partial x^{2}}+\frac{\partial^{2} v}{\partial y^{2}}\right) \\
& \rho_{f} u \frac{\partial w}{\partial x}+\rho_{f} v \frac{\partial w}{\partial y}=-\frac{\partial p}{\partial z}+\mu_{f}\left(\frac{\partial^{2} w}{\partial x^{2}}+\frac{\partial^{2} w}{\partial y^{2}}\right)
\end{aligned}
$$

where $u$ and $v$ are fluid velocities in $x$ and $y$ directions respectively, $p$ is pressure, and $\rho_{f}$ and $\mu_{f}$ are density and viscosity of liquid metal respectively. $f_{x}$ and $f_{y}$ are the electromagnetic forces acting on a unit volume metal in $x$ and $y$ directions respectively and obtained by solving magnetic field diffusion equation. ${ }^{11)}$

Equations (4), (5) and (6) were first solved using the finite volume method based on the SIMPLER method ${ }^{14)}$ to obtain velocity and pressure distribution in the cross section. Equation (7) was then solved to compute the axial velocity at a given axial pressure gradient, $\partial p / \partial z$, which was calibrated according to the average axial velocity. For a same average axial velocity, the small multichannel has higher pressure loss than the single-square channel due to increased friction force. Therefore, the value of $\partial p / \partial z$ was increased accordingly in order to obtain the same average axial velocity (i.e. flow rate) as the single-square channel in the case of multichannel.

The particle concentrations are governed by ${ }^{15)}$

$$
\frac{\partial u_{p} C}{\partial x}+\frac{\partial v_{p} C}{\partial y}+\frac{\partial w_{p} C}{\partial z}=0
$$

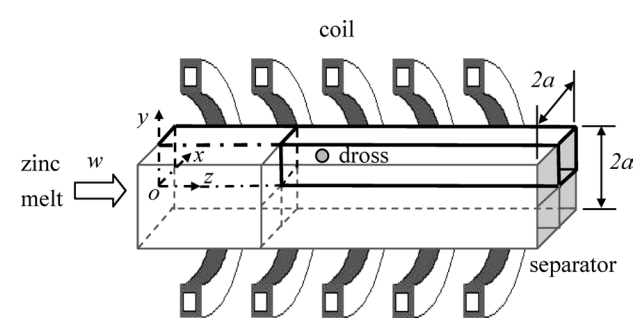

Fig. 2. The computational domain for the two-stage separator. 
where $C$ is the dimensionless particle concentration, and the velocities of particle migration, $u_{p}, v_{p}$ and $w_{p}$ in a magnetic field are expressed by:

$$
u_{p}=u-\frac{d_{p}^{2}}{24 \mu_{f}} f_{x}, \quad v_{p}=v-\frac{d_{p}^{2}}{24 \mu_{f}} f_{x}, \quad w_{p}=w
$$

where $d_{p}$ is particle diameter.

Because of the upwind nature of particle concentration, the particle concentrations in each cell at $z+\Delta z$ were obtained simultaneously by solving the discretised form of Eq. (8) using the values at $z$ by SOR method. ${ }^{16)}$ The initial conditions were $C=1$ at $z=0$ for the large channel and the calculated particle concentrations at the outlet of the large channel for the inlet of sub-channel at the rear.

The particle separation efficiency as a function of $z$ is determined from the net flux of particles flowing in and out of the cross-section of the channel:

$$
\eta=\left(1-\frac{1}{a^{2}} \int_{0}^{a} \int_{0}^{a} W C d x d y\right) \times 100 \%
$$

where $W=w / \bar{w}$ is a dimensionless axial velocity, and $\bar{w}$ is the average axial velocity.

For a tractable numerical solution, the following assumptions were made:

(1) The reduction in cross-section area from the singlesquare channel to the multi-square sub-channels was neglected so that $\bar{w}$ was constant in the multichannel; (2) the electromagnetic field was not affected by fluid flow because of small magnetic Reynolds number; (3) the particles were assumed non-conducting solid spheres and stopped immediately when reached the channel wall.

The physical properties of the $\mathrm{Zn}$ melt used in the simulation are listed in Table 1. Other parameters used in calculations are: the effective magnetic flux density, $B_{e}=0.05 \mathrm{~T}$; the frequency of magnetic field, $f=17.5 \mathrm{kHz}$; channel size, $a=5 \mathrm{~mm}$; particle diameters, $d_{p}=10,15$ and $20 \mu \mathrm{m}$.
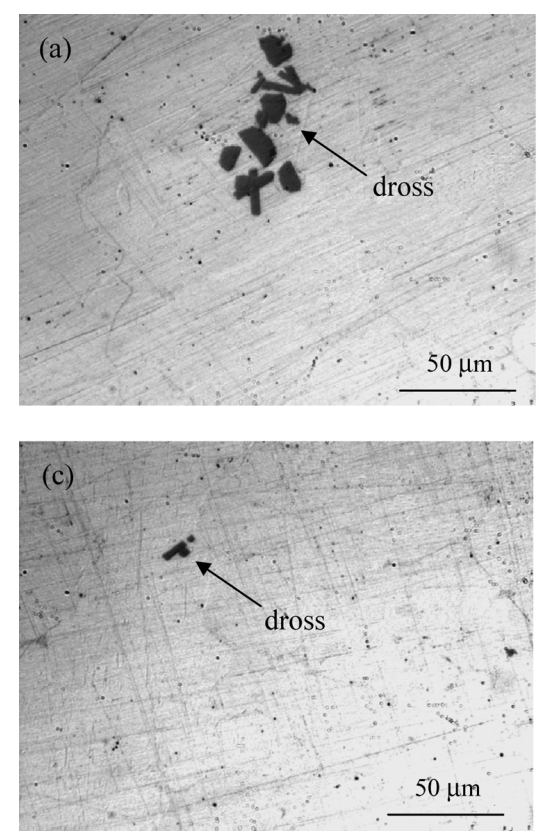

(d)

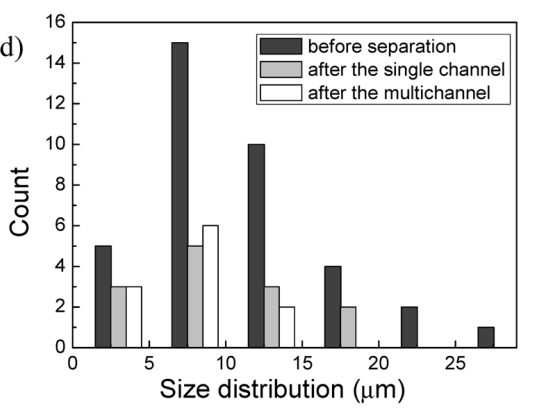

Fig. 3. Metallographic photos for samples and the measured size distribution of dross particles; a) before separation, b) after the single channel, c) after the two-stage multichannel, d) size distribution of the dross particles. 

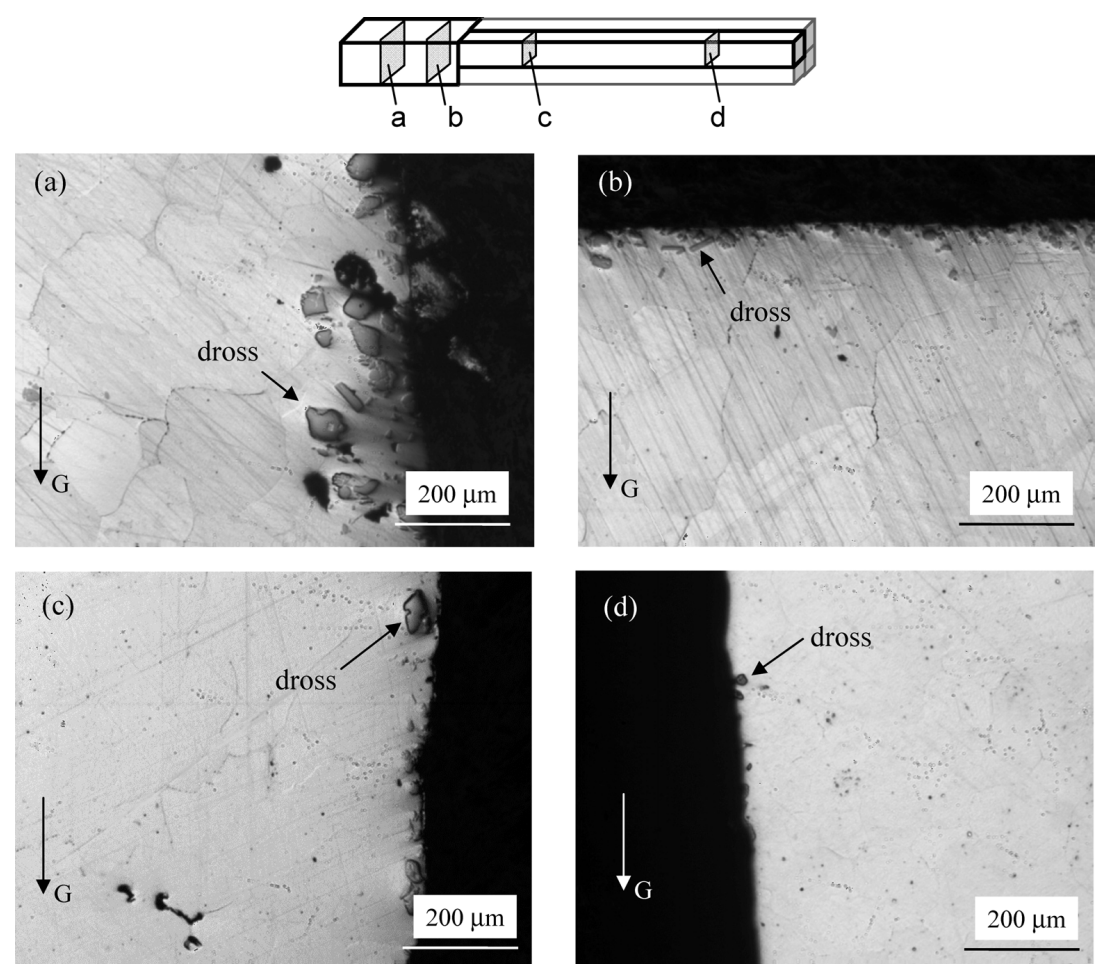

Fig. 4. Metallographic photos for samples of the residual taken from different sections in the multichannel separator; a) $z=20 \mathrm{~mm}, \mathrm{~b}$ ) $z=40 \mathrm{~mm}, \mathrm{c}) z=80 \mathrm{~mm}, \mathrm{~d}$ ) $z=160 \mathrm{~mm}$. (The arrow marked with $\mathrm{G}$ indicates the gravitational direction and the white and black parts denote the $\mathrm{Zn}$ matrix and surrounding resin respectively.)

mostly separated to the boundary at the front large channel (Fig. 4(a) and Fig. 4(b)), but a few were also captured by the sub-channel at the rear (Fig. 4(c)) and only minor small particles were captured near the outlet of the multichannel (Fig. 4(d)).

Table 3 gives the measured average area fraction and equivalent diameter of particles before and after single or multichannel separation. The separation efficiencies of the single channel and the multichannel based on image analysis are $77.4 \%$ and $87.3 \%$ respectively, close to those determined from chemical analysis. In conclusion, experimental results demonstrate an increase of about $10 \%$ in separation efficiency achieved when a multichannel was used instead of a single channel, despite that the electromagnetic parameters and flow conditions were nearly the same in both cases.

Calculations of particle separation efficiency $\eta$ versus separation time $t$ (the average residence time of melt flowing through the separator) are shown in Fig. 5, where the separation time for the large square channel of the multichannel was $1.5 \mathrm{~s}$ corresponding to the experimental condition. The separation efficiency is predicted to have a sharp increase of $10-15 \%$ as liquid metal flowed into the secondstage sub-channel, which matches well with the experiments.

Figure 6 compares the electromagnetic force density acting on liquid metal inside the front large channel and the sub-channel. Note that very weak force densities appear in the centre of the large channel due to the skin effect of alternating magnetic field (Fig. 6(a)), while this region in the sub-channel is subject to intense Lorentz force owing to the added walls (Fig. 6(b)). However, the magnitude of the Lorentz force decreases due to the reduced size of the sub-
Table 3. Image analysis of the specimens before and after separation.

\begin{tabular}{cccc}
\hline Specimens & $\begin{array}{c}\text { Average particle } \\
\text { diameter }(\mu \mathrm{m})\end{array}$ & $\begin{array}{c}\text { Average area fraction } \\
\text { of dross particles (\%) }\end{array}$ & $\begin{array}{c}\text { Separation } \\
\text { efficiency } \eta_{l}(\%)\end{array}$ \\
\hline Before separation & 10.7 & 0.283 & - \\
After the single channel & 8.9 & 0.064 & 77.4 \\
After the multichannel & 7.4 & 0.036 & 87.3 \\
\hline
\end{tabular}

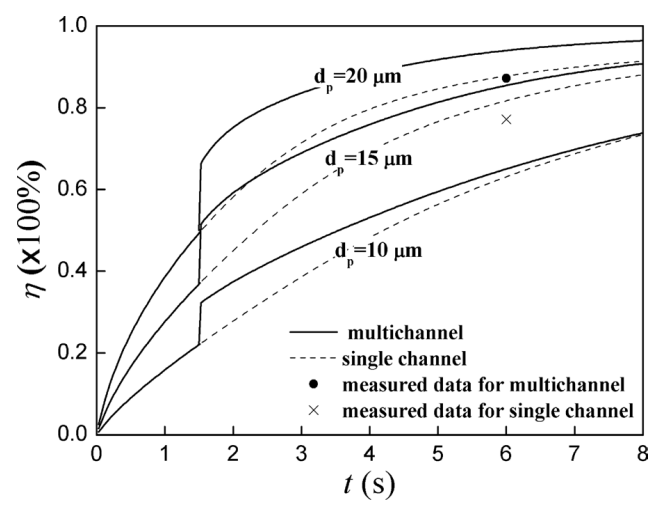

Fig. 5. Calculated separation efficiency as a function of separation time for the single-channel and the multichannel (experimental results are also shown in symbols).

channel.

The change in spatial distribution of the electromagnetic force density directly influences the particle migration behaviour. Based on the electromagnetic separation theory, ${ }^{17}$ a non-conducting particle is subject to an expulsive force in 
(a)

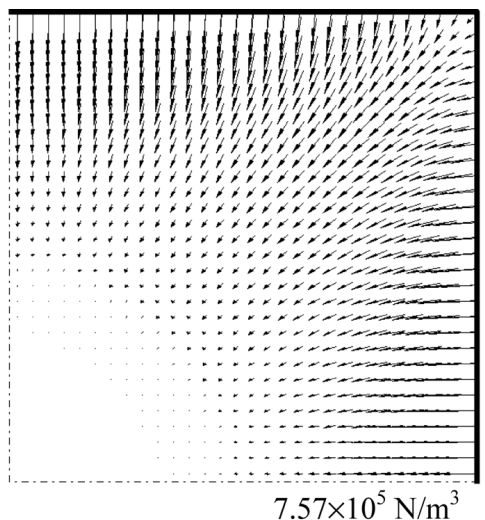

(b)

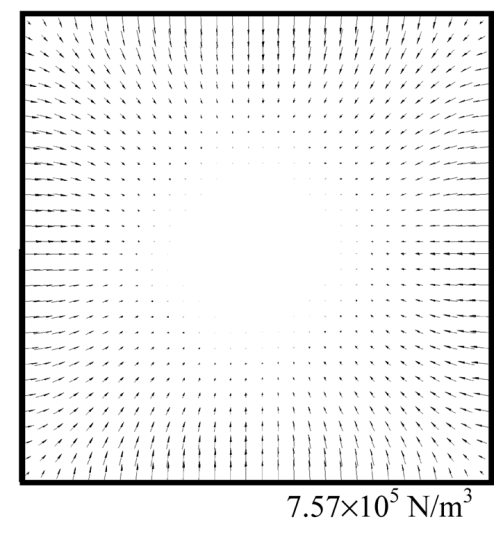

Fig. 6. Distribution of electromagnetic force density acting on the liquid metal; a) in the large single-channel, b) in the sub-channel.

(a)
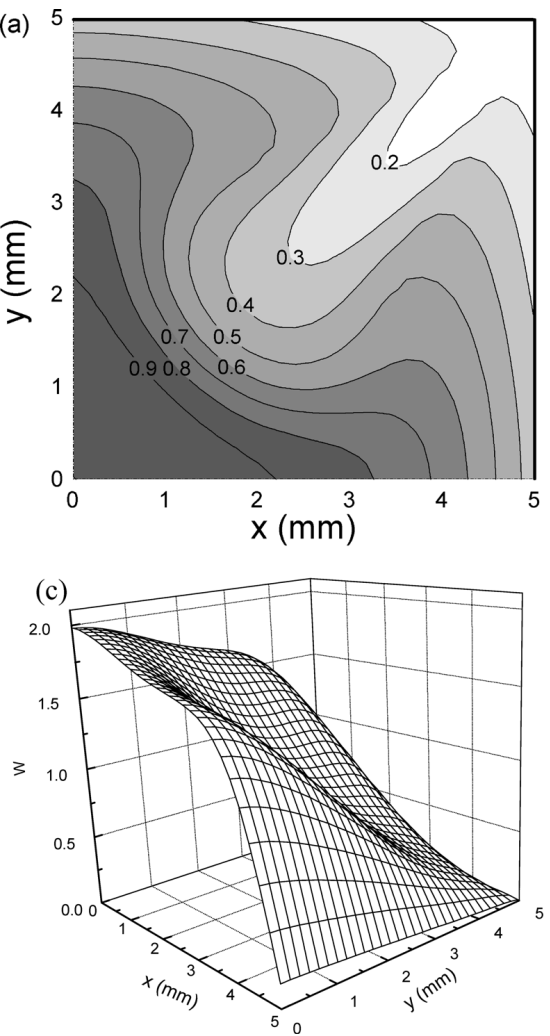

(b)
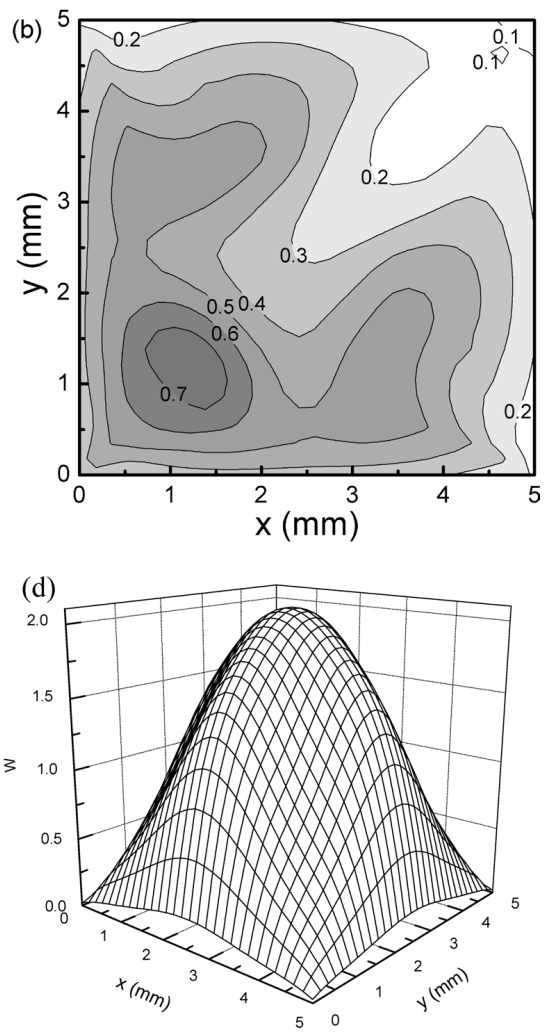

Fig. 7. Distributions of particle concentration $\left(d_{p}=15 \mu \mathrm{m}\right)$ and axial fluid velocity in the single-square channel and subchannel; a) particle distribution in the single-channel $(z=50 \mathrm{~mm})$, b) particle distribution in the sub-channel $(z=66 \mathrm{~mm}), \mathrm{c})$ axial velocity profile in the single-channel, d) axial velocity profile in the sub-channel.

an opposite direction and with a magnitude proportional to the Lorentz force acting on an equivalent volume liquid. As a result, particles in regions with high electromagnetic force densities migrate towards the walls more easily than those in regions with low electromagnetic force densities. Figure 7 shows the calculated concentration profiles at the end of the large single-square channel $(z=50 \mathrm{~mm})$ and shortly after entering the sub-channel $(z=66 \mathrm{~mm})$ for particles of $15 \mu \mathrm{m}$ in diameter respectively. Particle concentrations are higher in the inner region and lower near the wall in the single-square channel (Fig. 7(a)), which is exactly resulted from the unique distribution of Lorentz force density as shown in Fig. 6(a). However, particle concentrations in the inner region decrease significantly when the melt entered the multichannel (Fig. 7(b)), corresponding to the change in Lorentz force density as shown in Fig. 6(b).

Obviously, the change in concentration profiles helps improve the separation efficiency. However, it cannot account for the sharp increase in efficiency at the transition from the large channel to the sub-channel as shown in Fig. 5, because such change should occur gradually. Based on Eq. (10), the particle separation efficiency is a result of the net flux of particles flowing in and out of the cross-section of the channel, which is a product of particle concentration and flow rate. Therefore, any change in flow rate may influence the separation efficiency as well. Figure 7(c) and Fig. 7(d) show the dimensionless axial velocity distributions for the single-square channel and sub-channel respectively. Because of the drag effect of the added walls of the sub-channel, the velocity distribution changed significantly. The 
maximum velocity at $o z$ axis in the large single channel reduced to zero when the liquid flowed in the sub-channel. Note that the region containing high particle concentrations is exactly the one possessing high axial velocities for the large single-square channel. Therefore, it is the sudden decrease of flow rate in this area that leads to a sharp increase in the separation efficiency. The decrease of flow rate means the increase in the residence time, i.e. the particles have much longer time to migrate towards the wall. Apparently, this computed sudden jump was mainly because of the assumption made in the numerical model, and in reality, it is supposed to be a very short transient period involving an abrupt change in both particle velocity and concentration. A 3D model is currently under development to fully understand this transient process.

Nevertheless, from the current study, the main contribution to the increase in separation efficiency for the multichannel separator is the sharp decrease in the axial velocity around the inner region where the bulk melt with higher particle loading in the inner region of the first-stage channel was slowed dramatically when entered the sub-channel and the particles were naturally close to the channel walls and therefore have a shorter distance to travel to the walls. During flowing through the sub-channel, the melt maintained the similar velocity profile and particle concentration decreased gradually, resulting in a steady increase in the separation efficiency. Apparently it is the transition from the single channel to sub-channel that contributes significantly to the increase of the overall separation efficiency. A multistage and multichannel arrangement is therefore recommended for further increase in the separation efficiency of an electromagnetic separator. The difference between this new arrangement and the separator using a bundle of small channels presented by Yamao et al. ${ }^{10)}$ is that every channel has one or several sub-channels in the direction of fluid flow and is no longer a simple straight-pass one.

The measured separation efficiencies for both singlechannel and multi-channel based on image analysis that included the whole range of dross particle size $(0-30 \mu \mathrm{m})$ are also shown in Fig. 5. The measured values are approximate to the calculations for particles of about $15 \mu \mathrm{m}$, although the average particle diameter before separation is $10.7 \mu \mathrm{m}$ as given in Table 3. This may be attributed to the measured separation efficiency as a function of area fraction of particles, where larger particles contribute a large part to the total area fraction of particles and hence the separation of larger particles contributes more to the overall separation efficiency as well.

\section{Conclusions}

A two stage multichannel separator system was designed and investigated to increase the separation efficiency of non-metallic inclusions from bulk melt in an alternating magnetic field. Numerical method for calculating the particle concentration and separation efficiency is described and verified by experiments. Experimental results demonstrate that an increase of about $10 \%$ in separation efficiency can be achieved when a multichannel was used instead of a single channel. Calculations show that the distribution of electromagnetic force density and particle concentration change significantly due to the adding walls in the sub-channel. The separation efficiency increases abruptly when the melt flows through the single-channel into sub-channels, which is mainly attributed to the sharp decrease in axial velocity of the bulk melt with higher particle concentrations. A multistage and multichannel arrangement is therefore recommended for further increase in the separation efficiency of an electromagnetic separator.

\section{Acknowledgements}

The financial support from the National Basic Research Program of China (No. 2005CB623703), National Natural Science Foundation of China (Nos. 50825401\&50821003) and Royal Society International Joint Projects 2007/R4 of UK is gratefully acknowledged.

\section{REFERENCES}

1) L. Liu and F. H. Samuel: J. Mater. Sci., 33 (1998), 2269

2) H. Toda, T. Kobayashi and A. Takahashi: Mater. Sci. Eng. A, 280 (2000), 69.

3) N. El-Kaddah, A. D. Patel and T. T. Natarajan: JOM, 47 (1995), 46.

4) J. Park, A. Morihira, K. Sassa and S. Asai: Tetsu-to-Hagané, 80 (1994), 31

5) K. Takahashi and S. Taniguchi: ISIJ Int., 43 (2003), 820.

6) J. Park, K. Sassa and S. Asai: Nippon Kinzoku Gakkaishi, 59 (1995), 312.

7) K. Li, J. Wang, D. Shu, B. D. Sun and Y. H. Zhou: J. Mater. Sci. Technol., 18 (2002), 385.

8) A. P. Dong, D. Shu, J. Wang, X. C. Cai, B. D. Sun, J. Cui, J. G. Shen, Y. S. Ren and X. D. Yin: Mater. Sci. Technol., 24 (2008), 40.

9) A. P. Dong, D. Shu, J. Wang, B. D. Sun, J. G. Shen, Y. S. Ren, Y. L. Lu and X. D. Yin: Ironmaking Steelmaking, 36 (2009), 316.

10) F. Yamao, K. Sassa, K. Iwai and S. Asai: Tetsu-to-Hagané, 83 (1997), 30

11) D. Shu, B. D. Sun, K. Li and Y. H. Zhou: Scr. Mater, 48 (2003), 1385.

12) A. R. Marder: Prog. Mater. Sci., 45 (2000), 191.

13) S. Taniguchi and J. K. Brimacombe: ISIJ Int., 34 (1994), 722.

14) S. V. Patankar: Numerical Heat Transfer and Fluid Flow, Hemisphere Pub., McGraw-Hill, Washington, New York, (1980), 113.

$15)$ D. Shu, B. D. Sun, K. Li, J. Wang and Y. H. Zhou: ISIJ Int., 42 (2002), 1241.

16) W. H. Press, S. A. Teukolsky, W. T. Vetterling and B. P. Flannery: Numerical Recipes in C: The Art of Scientific Computing, 2nd ed., Cambridge University Press, Cambridge, (1992), 866.

17) D. Leenov and A. Kolin: J. Chem. Phys., 22 (1954), 683. 